\title{
Quantum Simulation of the Universal Features of the Polyakov Loop
}

\author{
Jin Zhang, ${ }^{1}$ J. Unmuth-Yockey, ${ }^{2}$ J. Zeiher, ${ }^{3}$ A. Bazavov, ${ }^{4}$ S.-W. Tsai, ${ }^{1}$ and Y. Meurice ${ }^{5}$ \\ ${ }^{1}$ Department of Physics and Astronomy, University of California, Riverside, California 92521, USA \\ ${ }^{2}$ Department of Physics, Syracuse University, Syracuse, New York 13244, USA \\ ${ }^{3}$ Max-Planck-Institut für Quantenoptik, 85748 Garching, Germany \\ ${ }^{4}$ Department of Computational Mathematics, Science and Engineering, and Department of Physics and Astronomy, \\ Michigan State University, East Lansing, Michigan 48824, USA \\ ${ }^{5}$ Department of Physics and Astronomy, The University of Iowa, Iowa City, Iowa 52242, USA
}

(Received 11 May 2018; revised manuscript received 16 October 2018; published 28 November 2018)

\begin{abstract}
Lattice gauge theories are fundamental to our understanding of high-energy physics. Nevertheless, the search for suitable platforms for their quantum simulation has proven difficult. We show that the Abelian Higgs model in $1+1$ dimensions is a prime candidate for an experimental quantum simulation of a lattice gauge theory. To this end, we use a discrete tensor reformulation to smoothly connect the space-time isotropic version used in most numerical lattice simulations to the continuous-time limit corresponding to the Hamiltonian formulation. The eigenstates of the Hamiltonian are neutral for periodic boundary conditions, but we probe the nonzero charge sectors by introducing either a Polyakov loop or an external electric field. In both cases we obtain universal functions relating the mass gap, the gauge coupling, and the spatial size, which are invariant under the deformation of the temporal lattice spacing. We propose to use a physical multileg ladder of atoms trapped in optical lattices and interacting with Rydberg-dressed interactions to quantum simulate the model and check the universal features. Our results provide a path to the analog quantum simulation of lattice gauge theories with atoms in optical lattices.
\end{abstract}

DOI: $10.1103 /$ PhysRevLett.121.223201

Lattice gauge theories (LGTs) are fundamental to our understanding of strongly interacting particles in highenergy physics. Translating the success of quantum simulations with cold atoms in optical lattices [1] of systems relevant to condensed matter physics, such as the BoseHubbard model, to the quantum simulation of LGTs would open the door to real-time and finite-density calculations, which are beyond the realm of classical computations. An important first step is to achieve this goal for models in one space and one time $(1+1)$ dimensions in a well-controlled setting. While the dynamics of the Schwinger model, quantum electrodynamics in $1+1$ dimensions, has been explored using a few qubit digital quantum simulation in a system of trapped ions [2] or classical-quantum algorithms on IBM quantum computers [3], the analog quantum simulation of gauge theories with cold atoms requires complex experimental settings. Existing efforts involve mixtures of bosonic and fermionic atoms [4,5] or dipolar interactions of cold molecules [6] and are still in progress.

In this Letter, we propose an experimental platform suited to quantum simulate the Abelian Higgs model in $1+1$

Published by the American Physical Society under the terms of the Creative Commons Attribution 4.0 International license. Further distribution of this work must maintain attribution to the author(s) and the published article's title, journal citation, and DOI. Funded by SCOAP. dimensions, the Schwinger model with the electron replaced by a complex scalar field. In particular, we envision probing the universal features of this model via a "Polyakov loop (PL)," a noncontractible Wilson loop wrapping around the periodic Euclidean time. In gauge theories, the expectation values of Wilson loops play a fundamental role, serving, for example, as order parameters to discriminate confined and deconfined phases of quarks and gluons in QCD. The Polyakov loop itself is relevant in finite-temperature studies [7]; however, its calculation at finite density with classical computing methods is often plagued by sign problems.

In contrast to other approaches [4-6,8-17] and similar to Ref. [2], we use a manifestly gauge-invariant formulation [18] where Gauss's law is inherently fulfilled. In addition, we consider the limit [18] where the scalar self-coupling becomes large and the Higgs mode decouples from the lowenergy theory. We are then left with a gauged $\mathrm{O}(2)$ spin model with compact field integration. Fourier analysis provides a discrete reformulation in agreement with Pontryagin duality [19] and suitable for an analog quantum simulation. For the experimental implementation, we invoke a single atomic species in an optical lattice on a multileg ladder and recently explored Rydberg-dressed interactions in this platform [20], aiming at maximal simplicity on both the theoretical and experimental side.

The remainder of the Letter is structured as follows. As a first result, we show novel numerical finite-size scaling 


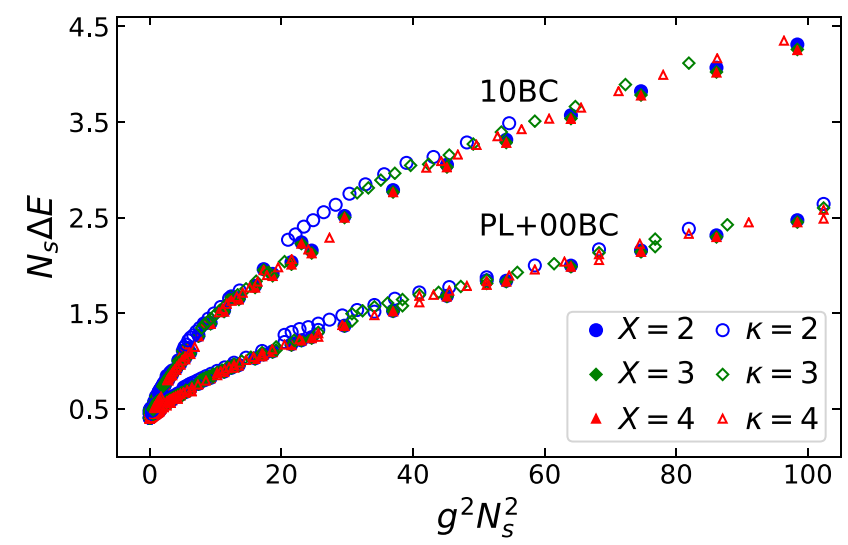

FIG. 1. $N_{s} \Delta E$ versus $g^{2} N_{s}^{2}$ for the gap $\Delta E$ created by the insertion of the Polaykov loop (lower set) or an external electric field (10 boundary conditions, upper set). Open (filled) markers represent Lagrangian (Hamiltonian) data. The choices of parameters, units, and methods for both of the 24 data sets are explained in the text.

(FSS) results for the Polyakov loop. This is followed by the derivation of the gauged $\mathrm{O}(2)$ spin Hamiltonian representing the Abelian Higgs model and the presentation of a blueprint for its experimental implementation. We conclude by outlining a strategy to benchmark the concepts of finitesize scaling in the Ising model as a simpler experimental setting, but with the essential experimental building blocks present.

The remarkable FSS properties of the Polyakov loop in the Abelian Higgs model are illustrated by the collapse of 24 data sets in Fig. 1. There are four different spatial sizes $N_{s}$ represented in the figure $\left(N_{s}=4,8,16\right.$, and 32), and it is possible to probe the critical behavior with systems of modest spatial sizes. We first give some brief explanations about these results and provide the details later in the text. The FSS is related to the energy gap $\Delta E$ created by inserting a Polyakov loop or by applying an external electric field. When the gauge coupling $g$ approaches zero, we have an $\mathrm{O}(2)$ model whose universal properties are of the Berezinsky-Kosterlitz-Thouless (BKT) type. The data collapse can be interpreted as originating from a relevant renormalization-group direction coming from the BKT conformal fixed point. When the hopping parameters exceed their critical value at the BKT transition, we have infinite correlation length at infinite volume and $\Delta E \propto$ $1 / N_{s}$ at finite $N_{s}$. Figure 1 indicates that when we turn on $g$, $N_{s} \Delta E$ is a linear function of $\left(g N_{s}\right)^{2}$ at small argument and then a linear function of $g N_{s}$ at larger argument. The two parts of Fig. 1 each contain 24 data sets with 12 from a discrete Euclidean time Lagrangian, while the other 12 are from the continuous-time Hamiltonian limit. It is remarkable that the two calculations provide the same universal functions. Ultimately, it is this equivalence between the two formulations which enables the transfer of results from the experimentally accessible Hamiltonian dynamics to the
Lagrangian formulation. Moreover, the FSS properties of the Polyakov loop allow for a well-controlled benchmark of an experimental implementation by transiting from small to large system sizes.

The considered Abelian Higgs model is described by the lattice path integral $Z=\int D \phi^{\dagger} D \phi D U e^{-S}$ with action

$$
\begin{aligned}
S= & -\beta_{p l} \sum_{x} \sum_{\nu<\mu} \operatorname{Re}\left[U_{x, \mu \nu}\right] \\
& -\kappa \sum_{x} \sum_{\nu=1}^{2}\left[\phi_{x}^{\dagger} U_{x, \nu} \phi_{x+\hat{\nu}}+\phi_{x+\hat{\nu}}^{\dagger} U_{x, \nu}^{\dagger} \phi_{x}\right] .
\end{aligned}
$$

The complex (charged) scalar field is $\phi_{x}=e^{i \theta_{x}}$ on spacetime sites $x$ and the Abelian gauge fields $U_{x, \mu}=e^{i A_{\mu}(x)}$ on the links from $x$ to $x+\hat{\mu}$. The electromagnetic tensor $F_{\mu \nu}=\partial_{\mu} A_{\nu}-\partial_{\nu} A_{\mu}$ appears when taking products of gauge fields around an elementary square (plaquette) in the $\mu \nu$ plane. The notation for this product is $U_{x, \mu \nu}=$ $e^{i\left[A_{\mu}(x)+A_{\nu}(x+\hat{\mu})-A_{\mu}(x+\hat{\nu})-A_{\nu}(x)\right]}$ and the gauge coupling enters through $\beta_{p l}=1 / g^{2}$. The hopping coefficient $\kappa$ can be asymmetric, and below we use $\kappa_{\tau}$ for the Euclidean time direction and $\kappa_{s}$ for the spatial direction.

The Fourier expansions of the Boltzmann weights lead to expressions of the partition function in terms of discrete sums of products of modified Bessel functions with integer orders on each plaquette and each link of the square lattice. The integer plaquette quantum numbers completely determine the integer link quantum numbers, which are the difference of the neighboring plaquette quantum numbers which can be interpreted as dual variables. Explicit formulas and sign conventions are given in Ref. [18], where we also show that the discrete tensor renormalization-group (TRG) [21,22] approach and the standard Monte Carlo approach give consistent numerical answers. The link quantum numbers can be interpreted as matter charges and their sum on the time links between two successive time slices stay constant as time is increased and define a conserved charge $Q$. When periodic boundary conditions, or open boundary conditions with zeros taken on the boundaries $(00 \mathrm{BC})$, are imposed for the gauge degrees of freedom, the condition $Q=0$ is automatically enforced.

The $Q \neq 0$ sectors can be probed by inserting a product $P$, called the Polyakov loop, of gauge links in the Euclidean time direction $\hat{\tau}$ with periodic boundary conditions:

$$
P=\prod_{n=0}^{N_{\tau}-1} U_{x^{*}+n \hat{\tau}, \hat{\tau}},
$$

inside the path integral. This is illustrated in Fig. 2. Conventional Monte Carlo simulations and TRG calculations with a typical bond dimension of $D_{\text {bond }} \approx 40$ provide consistent evidence [23] that $P$ decays exponentially with the size of the time domain $N_{\tau}$ : 


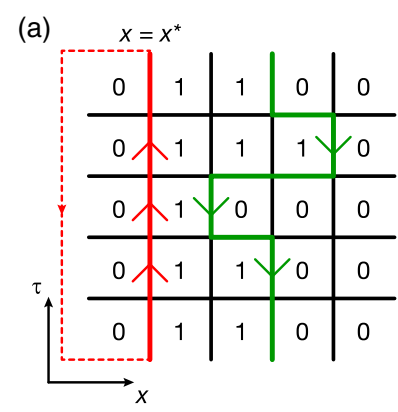

(b)

\begin{tabular}{l|l|l|l|l}
1 & 1 & 1 & 0 & 0 \\
\hline 1 & 1 & 1 & 1 & 0 \\
\hline 1 & 1 & 0 & 0 & 0 \\
\hline 1 & 1 & 1 & 0 & 0 \\
\hline 1 & 1 & 1 & 0 & 0
\end{tabular}

FIG. 2. The Polyakov loop (arrows pointing up) and the matter loop (arrows pointing down) composed of matter charges and plaquette quantum numbers for (a) 00BC and (b) 10BC without the Polyakov loop. Additional columns of zeros or ones are implicit. The dotted line indicates the wrapping in the Euclidean time direction.

$$
\langle P\rangle \propto e^{-N_{\tau} \Delta E} .
$$

$\langle P\rangle$ can be interpreted in terms of the free energy induced by the inclusion of a static charge. A similar effect can be induced by using asymmetric boundary conditions such as 1 on one side and 0 on the other side for the plaquette quantum numbers. This situation can be interpreted as the introduction of an external electric field and is denoted as $10 \mathrm{BC}$ in Fig. 1 and hereafter. With 00BC, translation invariance is not exact and the results depend on the location of the Polyakov loop $x^{\star}$. The $x^{\star}$ dependence and the possibility of obtaining $10 \mathrm{BC}$ by moving $x^{\star}$ to the boundary will be discussed in Ref. [23].

In order to connect the LGT calculations in the Lagrangian formulation with quantum simulations using the Hamiltonian formulation, we need to take the time continuum limit. This is done [18] by taking $\kappa_{\tau}, \beta_{p l} \rightarrow \infty$ while simultaneously taking $\kappa_{s}, a \rightarrow 0$ ( $a$ is the temporal lattice spacing) such that the combinations

$$
U \equiv \frac{1}{\beta_{p l} a}=\frac{g^{2}}{a}, \quad Y \equiv \frac{1}{2 \kappa_{\tau} a}, \quad X \equiv \frac{2 \kappa_{s}}{a}
$$

are kept constant. Note that $X$ here is related to $\tilde{X}$ in Ref. [18] by $X=\sqrt{2} \tilde{X}$. In this limit, using the properties of the Bessel functions, the Hamiltonian can be identified as a quantum rotor model and reads

$H=\frac{U}{2} \sum_{i=1}^{N_{s}}\left(L_{i}^{z}\right)^{2}+\frac{Y}{2} \sum_{i}^{\prime}\left(L_{i+1}^{z}-L_{i}^{z}\right)^{2}-X \sum_{i=1}^{N_{s}} U_{i}^{x}$.

The sum $\sum_{i}^{\prime}$, taking $00 \mathrm{BC}$ into account, includes $\left(L_{1}^{z}\right)^{2}+\left(L_{N_{s}}^{z}\right)^{2}$. The operator $U^{x}=\left(U^{+}+U^{-}\right) / 2$ with the special type of ladder operators $U^{+}, U^{-}$defined by $U^{ \pm}|m\rangle=|m \pm 1\rangle$, where $L^{z}|m\rangle=m|m\rangle$ with plaquette quantum numbers $m=0, \pm 1, \pm 2, \ldots$. If we truncate at $|m|_{\max }=s$, we call it a spin-s truncation even though it is different from the rotation group representation used in Ref. [24].

In the continuous-time limit, the introduction of the Polyakov loop amounts to changing the Hamiltonian into

$$
\begin{aligned}
\tilde{H}= & \frac{U}{2} \sum_{i=1}^{N_{s}}\left(L_{i}^{z}\right)^{2}+\frac{Y}{2} \sum_{i \neq N_{s} / 2}^{\prime}\left(L_{i+1}^{z}-L_{i}^{z}\right)^{2} \\
& +\frac{Y}{2}\left(L_{\left(N_{s} / 2\right)+1}^{z}-L_{N_{s} / 2}^{z}-1\right)^{2}-X \sum_{i=1}^{N_{s}} U_{i}^{x},
\end{aligned}
$$

where we have assumed that the Polyakov loop is put on the center of the lattice. The 10BC choice provides another way to probe the $Q \neq 0$ sector. This simply changes $\left(L_{1}^{z}\right)^{2}$ in the second summation of Eq. (5) to $\left(L_{1}^{z}-1\right)^{2}$.

The numerical continuous-time results were obtained using the density matrix renormalization group (DMRG) $[25,26]$ to calculate the ground state energies for both cases. The finite DMRG algorithm with matrix product state [27] optimization was performed using the ITensor $\mathrm{C}++$ library [28]. $Y=1$ units and a spin-6 truncation [23] were used in all DMRG calculations.

As explained in more detail in Ref. [23], arguments regarding the behavior at small and large $g N_{s}$ led us to conjecture that $N_{s} \Delta E$ is solely a function of the product $\left(g N_{s}\right)^{2}$. Figure 1 supports this idea and shows a good data collapse across multiple system sizes for both the discreteand continuous-time limits. Note that for the discrete-time (Lagrangian) calculations at various $\kappa, \Delta E$ was rescaled by $2 \kappa$, while for the continuous-time (Hamiltonian) calculations, a similar rescaling of $2 \kappa_{\tau} a$ was introduced by setting $Y=1$. We emphasize that the collapse is by no means automatic. It breaks down for $\kappa$ not large enough, if we increase $g$ to large values while keeping $N_{s}$ constant (there are hints of this in Fig. 1 for the small $N_{s}$ data), or if the truncation value is smaller than $m_{\max }=2$.

Notice that in all cases, $N_{s} \Delta E \simeq 0.5$ when $g^{2} \simeq 0$, which corresponds to the gapless BKT phase in the $\mathrm{O}(2)$ limit. We also notice that the energy gap at finite $g^{2}$ for $10 \mathrm{BC}$ is bigger than the gap for PL-00BC. Because 10BC breaks the inversion symmetry of the system, creating a charge on the right costs more energy than that on the left. We can understand this by doing the transformation $L_{i}^{\prime z}=L_{i}^{z}-1$ for $i \leq N_{s} / 2$. Then the Hamiltonian with 10BC is related to the Hamiltonian with the Polyakov loop by $H_{10} \rightarrow$ $\tilde{H}+U \sum_{i=1}^{N_{s} / 2} L_{i}^{\prime z}+N_{s} U / 4$, which is simply adding a linear potential on the left half of the system. But the data collapse is still present, indicating the same universality class.

An important feature of the Hamiltonians considered above is that $L_{i}^{z}$ has positive and negative eigenvalues and cannot be realized as the number operator of a Bose-Hubbard model unless a large chemical potential $[24,29,30]$ or two atomic species are introduced [24]. For a 


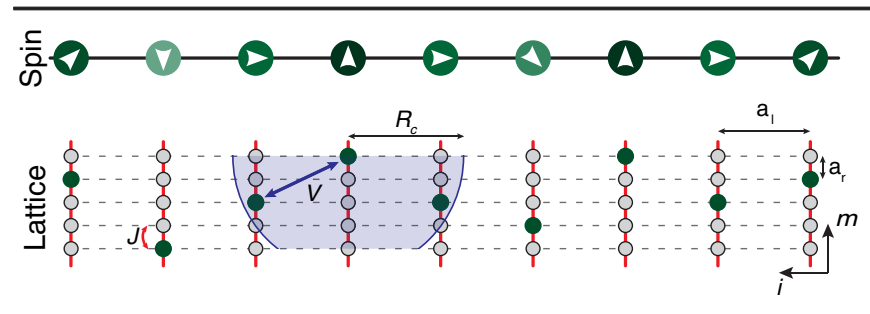

FIG. 3. Multileg ladder implementation for spin-2. The upper part shows the possible $m_{z}$ projections. Below, we show the corresponding realization in a ladder within an optical lattice. The atoms (green disks) are allowed to hop within a rung with a strength $J$, while no hopping is allowed along the legs. The lattice constants along rungs and legs are $a_{r}$ and $a_{l}$, respectively. Coupling between atoms in different rungs is implemented via an isotropic Rydberg-dressed interaction $V$ with a cutoff distance $R_{c}$ (marked by blue shading).

similar reason, a two-leg ladder with $2 s$ atoms per rung for a spin- $s$ truncation was suggested in Ref. [18]; however, the hopping along a rung can only emulate the $L^{x}$ operator in the rotation group representation instead of the $U^{x}$ operator in Eqs. (5) and (6). Here, we propose a simpler experimental realization to overcome this difficulty, namely an asymmetric ladder of $N_{s}$ rungs of length $2 s+1$ each with a single atom per rung. The lattice constants are $a_{l}$ and $a_{r}$ along legs and rungs, respectively, see Fig. 3, and the tunnel coupling along the legs is vanishing while it has a strength $J$ along the rungs. In this case, the $L^{z}$ projection of the spin is encoded in the position $m$ of the atom within a given rung and can be read out with near-unity fidelity in a quantum gas microscope [1]. The initialization of the system can be achieved in such a setup by preparing an atomic Mott insulator and employing site-resolved optical potentials [31].

We now aim at establishing a quantitative connection between the spin Hamiltonians Eqs. (5) and (6) and the Hamiltonian of such a ladder system. The latter reads

$$
\begin{aligned}
\hat{H}= & -\frac{J}{2} \sum_{i=1}^{N_{s}} \sum_{m=-s}^{s-1}\left(\hat{a}_{m, i}^{\dagger} \hat{a}_{m+1, i}+\text { H.c. }\right)-\sum_{i=1}^{N_{s}} \sum_{m=-s}^{s} \epsilon_{m, i} \hat{n}_{m, i} \\
& +\sum_{i, i^{\prime}=1}^{N_{s}} \sum_{m, m^{\prime}=-s}^{s} V_{m, m^{\prime}, i, i^{\prime}} \hat{n}_{m, i} \hat{n}_{m^{\prime}, i^{\prime}} .
\end{aligned}
$$

Here, we have introduced an interaction $V_{m, m^{\prime}, i, i^{\prime}}$ between two particles at positions $(m, i)$ and $\left(m^{\prime}, i^{\prime}\right)$ as well as an on-site potential $\epsilon_{m, i}$. The term $X \sum_{i} U_{i}^{x}$ in Eqs. (5) and (6) directly maps to the tunneling term in Hamiltonian Eq. (7) for $J=X$.

Realizing the other two terms requires fine-tuned values $V_{m, m^{\prime}, i, i^{\prime}}=V_{m, m^{\prime}} \delta_{i^{\prime}, i+1}$, with $V_{m, m^{\prime}}=-\left|V_{0}\right|+Y\left(m-m^{\prime}\right)^{2} / 2$ for the interaction potential between two particles constrained by the Kronecker symbol $\delta_{i^{\prime}, i+1}$ to be located in two neighboring rungs. Furthermore, the on-site potentials have to be tuned to $\epsilon_{m, i}=-U m^{2} / 2$ for the rungs with $i \neq 1$,

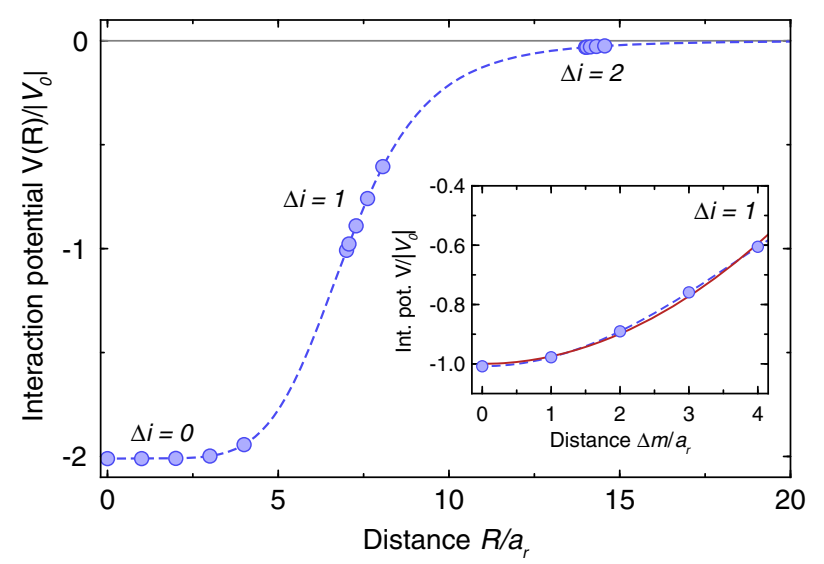

FIG. 4. Quadratic interactions on an asymmetric ladder for $s=2$. The isotropic Rydberg-dressed potential (dashed blue line) is sampled at different distances occurring in the ladder (blue points). Interactions between atoms in different rungs separated by $\Delta i=\left|i-i^{\prime}\right|$ occur in groups. The inset shows the approximate quadratic dependence for $\Delta i=1$ versus distance $\Delta m=\left|m-m^{\prime}\right|$ within a rung compared to a true quadratic interaction (red solid line). The parameters used are $R_{c}=a_{l}=7 a_{r}$.

$N_{s}$ and $\epsilon_{m, 1}=\epsilon_{m, N_{s}}=-(U+Y) m^{2} / 2$ for two rungs at the boundaries, up to an irrelevant constant energy offset $-\left(N_{s}-1\right)\left|V_{0}\right| / N_{s}$ implied for all on-site potentials.

Introducing a Polyakov loop amounts to changing the on-site potentials on the two central rungs $N_{s} / 2$ and $N_{s} / 2+1$ to $\epsilon_{m, N_{s} / 2}=-U m^{2} / 2-Y m$ and $\epsilon_{m, N_{s} / 2+1}=$ $-U m^{2} / 2+Y(m-1 / 2)$, respectively. The boundary condition $10 \mathrm{BC}$ can be realized by tuning the on-site potential at one end of the ladder to $\epsilon_{m, 1}=-U m^{2} / 2-Y(m-1)^{2} / 2$.

While the tailored on-site potentials $\epsilon_{m, i}$ can be generated using optical potentials controlled at the single-site level [32], realizing the quadratic distance dependence of the interaction between two particles is challenging in cold atomic gases. However, they can still be realized approximately using off-resonant optical coupling of the atoms to Rydberg states. The resulting isotropic Rydbergdressed interactions $[33,34]$ in cold atoms have recently begun to be explored in a many-body setting [20] and exhibit a characteristic distance dependence $V(R)=$ $U_{0} /\left[1+\left(R / R_{c}\right)^{6}\right]$ for two atoms separated by a distance $R$. The saturation value $U_{0}$ can be tuned to be positive or negative, and the interaction range $R_{c}$ is set by the interactions of the coupled Rydberg states and typically reaches up to several sites in an optical lattice [35].

The key idea in implementing quadratic interactions in the ladder model consists in utilizing an asymmetric ladder with different lattice constants along legs and rungs, respectively. In the limit of large $a_{l} / a_{r}$, the interaction potential along the rung approximately acquires the desired quadratic distance dependence for neighboring rungs with $\left|V_{0}\right|=\left|U_{0}\right| /\left[1+\left(a_{l} / R_{c}\right)^{6}\right]$ and $Y=6\left|U_{0}\right|\left(a_{l} / R_{c}\right)^{6}\left(a_{r} / a_{l}\right)^{2} /\left[1+\left(a_{l} / R_{c}\right)^{6}\right]^{2}$. At the same 


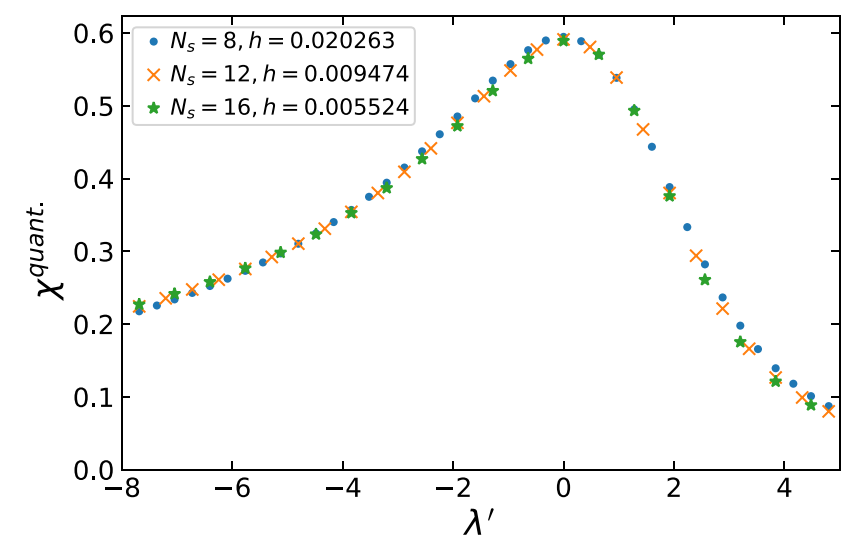

FIG. 5. Data collapse for the quantum magnetic susceptibility of the quantum Ising chain with the known rescaling $\chi^{\text {quant' }}=$ $\chi^{\text {quant }} N_{s}^{-(1-\eta)}$ versus $\lambda^{\prime}=N_{s}^{1 / \nu}(\lambda-1)$. The reduced magnetic field $h^{\prime}=h N_{s}^{15 / 8}=1$ for all three system sizes.

time, interactions between next-nearest-neighbor rungs can be minimized, see Fig. 4, making them irrelevant for the predicted collapse shown in Fig. 1. This and other imperfections as well as concrete experimental numbers are further discussed in Ref. [35].

A strength of the presented ladder implementation is the simple realization of models with different spin. A natural first step would be to check the experimental feasibility of the proposal with just two legs, i.e., $s=1 / 2$ in Eq. (7). The emerging spin model corresponds to the well-studied spin-1/2 quantum Ising chain in a transverse field with the Hamiltonian

$$
\hat{H}=-\lambda \sum_{i=1}^{N_{s}} \hat{\sigma}_{i}^{z} \hat{\sigma}_{i+1}^{z}-h_{x} \sum_{i=1}^{N_{s}} \hat{\sigma}_{i}^{x}-h \sum_{i=1}^{N_{s}} \hat{\sigma}_{i}^{z}
$$

The transverse field is realized by the tunneling of the atoms and has a strength $h_{x}=J / 2$. Tuning $\epsilon_{ \pm 1 / 2}=$ $\pm h-\left(N_{s}-1\right)\left(\left|V_{0}\right|-\lambda\right) / N_{s}, V_{1 / 2,1 / 2}=-\left|V_{0}\right|, V_{-1 / 2,1 / 2}=$ $-\left|V_{0}\right|+2 \lambda$ is required to realize the other two terms.

Expressing all energies in units of the transverse field $\left(h_{x}=1\right)$, this model has a second-order phase transition at $\lambda=1$ with known exponents [39]. As quantum simulations are still made on relatively small lattices, it is convenient to study the finite-size scaling dictated by the renormalization-group analysis of the second-order phase transition. The zero temperature magnetic susceptibility reads

$$
\chi^{\text {quant }}=\frac{1}{N_{s}} \sum_{\langle i, j\rangle}\left\langle\left(\hat{\sigma}_{i}^{z}-\left\langle\hat{\sigma}_{i}^{z}\right\rangle\right)\left(\hat{\sigma}_{j}^{z}-\left\langle\hat{\sigma}_{j}^{z}\right\rangle\right)\right\rangle,
$$

where $\langle\cdots\rangle$ are short notations for $\langle\Omega|\ldots| \Omega\rangle$ with $|\Omega\rangle$ the lowest energy state of $\hat{H}$. The data collapse obtained with the standard RG rescalings is illustrated in Fig. 5.
The quantum Ising model has, for example, been quantum simulated in systems of ultracold ions [40] and with atoms in tilted optical lattices [41]. New generations of $D$-wave machines have more versatile time-dependent capabilities. It seems possible to maintain a transverse field [42], but there are temperature effects that need to be better understood. Multimode cavity photon-mediated interactions [43] can also be used to simulate the quantum Ising model. The possibility of extending these setups or related ones to reproduce a multileg ladder is being investigated.

In conclusion, we have presented an experimental platform for the quantum simulation of the Abelian Higgs model in $1+1$ dimension and outlined a strategy for an initial benchmark of the quantum simulator. An interesting perspective is the experimental simulation of out-ofequilibrium dynamics following a quantum quench, which promises insight into dynamics described by the LGT when inaccessible with classical computing.

We thank I. Bloch, S. Catterall, and D. Lidar for valuable conversations. This work was supported in part by the U.S. Department of Energy (DOE) under Awards No. DE-SC0010113, No. DE-SC0019139 (Y.M.) and No. DE-SC0009998 (J. U.-Y.) and by the NSF under Grant No. DMR-1411345 (S.-W. T.). J. Z. was supported by MPG and the European Commission (UQUAM).

[1] C. Gross and I. Bloch, Science 357, 995 (2017).

[2] E. A. Martinez, C. A. Muschik, P. Schindler, D. Nigg, A. Erhard, M. Heyl, P. Hauke, M. Dalmonte, T. Monz, P. Zoller et al., Nature (London) 534, 516 (2016).

[3] N. Klco, E. F. Dumitrescu, A. J. McCaskey, T. D. Morris, R. C. Pooser, M. Sanz, E. Solano, P. Lougovski, and M. J. Savage, Phys. Rev. A 98, 032331 (2018).

[4] D. Banerjee, M. Dalmonte, M. Müller, E. Rico, P. Stebler, U.-J. Wiese, and P. Zoller, Phys. Rev. Lett. 109, 175302 (2012).

[5] V. Kasper, F. Hebenstreit, F. Jendrzejewski, M. K. Oberthaler, and J. Berges, New J. Phys. 19, 023030 (2017).

[6] B. Gadway, Proceedings of the NPQI meeting, Argonne, 2018, http://www.phy.anl.gov/npqi2018/talks/gadway.pdf.

[7] K. Fukushima and V. Skokov, Prog. Part. Nucl. Phys. 96, 154 (2017).

[8] E. Zohar and B. Reznik, Phys. Rev. Lett. 107, 275301 (2011).

[9] E. Zohar, J. I. Cirac, and B. Reznik, Phys. Rev. Lett. 109, 125302 (2012).

[10] L. Tagliacozzo, A. Celi, A. Zamora, and M. Lewenstein, Ann. Phys. (Amsterdam) 330, 160 (2013).

[11] E. Zohar, J. I. Cirac, and B. Reznik, Phys. Rev. Lett. 110, 125304 (2013).

[12] K. Kasamatsu, I. Ichinose, and T. Matsui, Phys. Rev. Lett. 111, 115303 (2013).

[13] U.-J. Wiese, Ann. Phys. (Berlin) 525, 777 (2013).

[14] E. Zohar, J. I. Cirac, and B. Reznik, Rep. Prog. Phys. 79, 014401 (2016). 
[15] Y. Kuno, K. Kasamatsu, Y. Takahashi, I. Ichinose, and T. Matsui, New J. Phys. 17, 063005 (2015).

[16] Y. Kuno, S. Sakane, K. Kasamatsu, I. Ichinose, and T. Matsui, Phys. Rev. D 95, 094507 (2017).

[17] D. Gonzalez-Cuadra, E. Zohar, and J. I. Cirac, New J. Phys. 19, 063038 (2017).

[18] A. Bazavov, Y. Meurice, S.-W. Tsai, J. Unmuth-Yockey, and J. Zhang, Phys. Rev. D 92, 076003 (2015).

[19] L. Pontryagin, Topological Groups (Princeton University, Princeton, 1939).

[20] J. Zeiher, R. van Bijnen, P. Schauß, S. Hild, J.-y. Choi, T. Pohl, I. Bloch, and C. Gross, Nat. Phys. 12, 1095 (2016).

[21] Z. Y. Xie, J. Chen, M. P. Qin, J. W. Zhu, L. P. Yang, and T. Xiang, Phys. Rev. B 86, 045139 (2012).

[22] Y. Liu, Y. Meurice, M. P. Qin, J. Unmuth-Yockey, T. Xiang, Z. Y. Xie, J. F. Yu, and H. Zou, Phys. Rev. D 88, 056005 (2013).

[23] J. Unmuth-Yockey, J. Zhang, A. Bazavov, Y. Meurice, and S.-W. Tsai, arXiv:1807.09186.

[24] H. Zou, Y. Liu, C.-Y. Lai, J. Unmuth-Yockey, L. P. Yang, A. Bazavov, Z. Y. Xie, T. Xiang, S. Chandrasekharan, S. W. Tsai, and Y. Meurice, Phys. Rev. A 90, 063603 (2014).

[25] S. R. White, Phys. Rev. Lett. 69, 2863 (1992).

[26] U. Schollwöck, Ann. Phys. (Amsterdam) 326, 96 (2011).

[27] S. Östlund and S. Rommer, Phys. Rev. Lett. 75, 3537 (1995).

[28] Version 2.1.1, http://itensor.org/.

[29] J. Unmuth-Yockey, J. Zhang, P. M. Preiss, L.-P. Yang, S. W. Tsai, and Y. Meurice, Phys. Rev. A 96, 023603 (2017).

[30] A. Bazavov, Y. Meurice, S. W. Tsai, J. Unmuth-Yockey, L.-P. Yang, and J. Zhang, Phys. Rev. D 96, 034514 (2017).
[31] C. Weitenberg, M. Endres, J. F. Sherson, M. Cheneau, P. Schauß, T. Fukuhara, I. Bloch, and S. Kuhr, Nature (London) 471, 319 (2011).

[32] T. Fukuhara, A. Kantian, M. Endres, M. Cheneau, P. Schauß, S. Hild, D. Bellem, U. Schollwöck, T. Giamarchi, C. Gross, I. Bloch, and S. Kuhr, Nat. Phys. 9, 235 (2013).

[33] N. Henkel, R. Nath, and T. Pohl, Phys. Rev. Lett. 104, 195302 (2010).

[34] G. Pupillo, A. Micheli, M. Boninsegni, I. Lesanovsky, and P. Zoller, Phys. Rev. Lett. 104, 223002 (2010).

[35] See Supplemental Material at http://link.aps.org/ supplemental/10.1103/PhysRevLett.121.223201, which additionally includes Refs. [36-38], for a discussion of experimental aspects of the quadratic interactions, measurement of the energy gap and numerical estimates.

[36] M. Endres, M. Cheneau, T. Fukuhara, C. Weitenberg, P. Schauß, C. Gross, L. Mazza, M. C. Bañuls, L. Pollet, I. Bloch, and S. Kuhr, Appl. Phys. B 113, 27 (2013).

[37] B. Santra, C. Baals, R. Labouvie, A. B. Bhattacherjee, A. Pelster, and H. Ott, Nat. Commun. 8, 15601 (2017).

[38] R. M. W. van Bijnen and T. Pohl, Phys. Rev. Lett. 114, 243002 (2015)

[39] P. Pfeuty, Ann. Phys. (N.Y.) 57, 79 (1970).

[40] R. Blatt and C. F. Roos, Nat. Phys. 8, 277 (2012).

[41] J. Simon, W. S. Bakr, R. Ma, M. E. Tai, P. M. Preiss, and M. Greiner, Nature (London) 472, 307 (2011).

[42] A. D. King et al., Nature (London) 560, 456 (2018).

[43] V. D. Vaidya, Y. Guo, R. M. Kroeze, K. E. Ballantine, A. J. Kollár, J. Keeling, and B. L. Lev, Phys. Rev. X 8, 011002 (2018). 